\title{
UNIFIED CONTACT STRUCTURE
}

\author{
B.B. SINHA AND R.N. YADAV
}

\begin{abstract}
A bstract. A necessary and sufficient condition that a $C^{\infty}$ manifold to admits anunified contact structure is established. A condition is obtained so that it becomes P-Sasakian. A linear connection in it is defined so its distributions are parallel.
\end{abstract}

\section{Introduction}

If, on a manifold $M$, there exists a tensor field $\phi$ of type $(1,1), r$ vector fields $\xi_{1}, \xi_{2}, \ldots, \xi_{r}$ and $r$-1-forms $\eta^{1}, \cdots, \eta^{r}$ such that

$$
\begin{gathered}
\eta^{\alpha}\left(\xi_{\beta}\right)=\delta_{\beta}^{\alpha}, \alpha, \beta \in(r)=\{1, \cdots, r\} \\
\phi^{2}=a^{2}\left\{I-\eta^{\alpha} \otimes \xi_{\alpha}\right\}
\end{gathered}
$$

where $a^{\alpha} b_{\alpha}$ denote $\sum a^{\alpha} b_{\alpha}$ where ' $a$ ' is a complex constant and $I$ denotes identity operator

$$
\begin{array}{ll}
\eta^{\alpha} \circ \phi=0, & \alpha \in(r) \\
\phi\left(\xi_{\alpha}\right)=0, & \alpha \in(r) .
\end{array}
$$

Then $\Sigma=\left(\phi, \xi_{\alpha}, \eta^{\alpha}\right)_{\alpha \in(r)}$ is called an unfield contact structure on $M$ and $M$ is an unified contact manifold.

If the structure $\phi$ is compatible with the Riemannian metric $g$, i.e.

$$
g(\phi X, \phi Y)=\left\{g(X, Y)-\sum_{\alpha} \eta^{\alpha}(X) \eta^{\alpha}(Y)\right\} .
$$

For any vectors $X$ and $Y$. Then $a^{2}= \pm 1$. In case of $a^{2}=-1$, the structure $\phi$ is almost contact and skew-symmetric. In case of $a^{2}=1$, the structure $\phi$ is almost paracontact and symmetric. If $\phi$ is parallel with respect to the Riemannian connection, then $(g, \phi)$ is Sasakian or para-contact structure respectively.

If one put the condition

$$
g(\phi X, \phi Y)=\lambda\left\{g(X, Y)-\sum_{\alpha} \eta^{\alpha}(X) \eta^{\alpha}(Y)\right\}
$$

Received January 5, 1988 
in place of the compatibility (1.5), then $\lambda$ is positive because the metric $g$ is positive definite, and $\lambda^{2}=a^{4}$ or $\lambda=\left|a^{2}\right|$ by means of (1.6) and the structure condition (1.2).

An unified contact manifold $M$ always admits the following complementary distributions.

$$
\begin{aligned}
D^{+} & =\{X ; \phi(X)=a X\}, \\
D^{-} & =\{X ; \phi(X)=-a X\}, \\
D^{0} & =\{X ; \phi(X)=0\},
\end{aligned}
$$

we define operators

$$
\begin{aligned}
& \phi_{1}=\frac{1}{2}\left\{I+\frac{1}{a} \phi-\eta^{\alpha} \otimes \xi_{\alpha}\right\}=\frac{1}{2}\left\{\frac{1}{a^{2}} \phi^{2}+\frac{1}{a} \phi\right\}, \\
& \phi_{2}=\frac{1}{2}\left\{I-\frac{1}{a} \phi-\eta^{\alpha} \otimes \xi_{\alpha}\right\}=\frac{1}{2}\left\{\frac{1}{a^{2}} \phi^{2}-\frac{1}{a} \phi\right\}, \\
& \phi_{3}=\left\{I-\frac{1}{a^{2}} \phi^{2}\right\},
\end{aligned}
$$

They have following properties

$$
\begin{gathered}
\phi_{1}+\phi_{2}+\phi_{3}=I, \\
\phi_{1}^{2}=\phi_{1}, \quad \phi_{2}^{2}=\phi_{2}, \quad \phi_{3}^{2}=\phi_{3}, \\
\phi_{1} \circ \phi_{2}=\phi_{2} \circ \phi_{1}=\phi_{1} \circ \phi_{3}=\phi_{3} \circ \phi_{1}=\phi_{2} \circ \phi_{3}=\phi_{3} \circ \phi_{2}=0
\end{gathered}
$$

and moreover,

$$
\begin{aligned}
& \phi \circ \phi_{1}=\phi_{1} \circ \phi=a \phi_{1}, \\
& \phi \circ \phi_{2}=\phi_{2} \circ \phi=-a \phi_{2}, \\
& \phi \circ \phi_{3}=\phi_{3} \circ \phi=0 .
\end{aligned}
$$
follows

By $(1.16),(1.17),(1.18)$ the distributions $D^{+}, D^{-}$and $D^{0}$ may be expressed as

$$
\begin{aligned}
& D^{+}=\left\{X ; \phi_{1} X=X\right\} \\
& D^{-}=\left\{X ; \phi_{2} X=X\right\} \\
& D^{0}=\left\{X ; \phi_{3} X=0\right\}
\end{aligned}
$$

Thus we have Lemma,

Lemmal 1 .[3]. The distributions $D^{+}, D^{-}, D^{0}$ are generated by the projection operators $\phi_{1}, \phi_{2}, \phi_{3}$ respectively. 
Theorem 1. The necessary and sufficient condition for $M$ admits an unified contact structure is that there exists three complementary distributions $D_{1}, D_{2}, D_{3}$ of dimension $p, q, r$ respectively, with $p+q+r=n=\operatorname{dim} M$.

Proof. The necessary condition immediately follows from Lemma 1 . Now suppose that there are three complementary distributions $D_{1}, D_{2}, D_{3}$ of dimension $p, q, r$ respectively, with $p+q+r=\operatorname{dim} M=n$.

For any $X \in M$, we have $T_{x} M=D_{1} x+D_{2} x+D_{3} x$. Let

$$
\left\{e_{1}, \ldots, e_{p}, e_{p+1}=\bar{e}_{1}, \ldots, e_{p+q}=\bar{e}_{q}, e_{p+q+1}=\xi_{1}, \ldots, e_{n}=\xi_{r}\right\}
$$

be a bașis of $T_{x} M$, where $\left\{e_{i} ; i \in(P)\right\}$ is a basis for $D_{1} x,\left\{\bar{e}_{t} ; t \in(q)\right\}$ is a basis for $D_{2} x$, and $\left\{\xi_{1}, \ldots, \xi_{r}\right\}$ is a basis for $D_{3} x$.

Now let $\left\{e^{1}, \cdots, e^{p}, e^{p+1}=\bar{e}^{-1}, \cdots, e^{p+q}=\bar{e}^{q}, e^{p+q+1}=\eta^{1}, \cdots, e^{n}=\eta^{r}\right\}$ be a basis of cotangent space $T_{x}^{*} M$ such that

$$
e^{i}\left(e_{j}\right)=\delta_{j}^{i}, \quad i, j \in(n)
$$

which gives,

$$
e^{k} \otimes e_{k}+\bar{e}^{t} \otimes \bar{e}_{t}+\eta^{\alpha} \otimes \xi_{\alpha}=I
$$

where

$$
k \in(p), \quad t \in(q), \quad \alpha \in(r) .
$$

Let us put

$$
\phi=a\left\{e^{k} \otimes e_{k}+\varepsilon \bar{e}^{t} \otimes \bar{e}_{t}\right\}, \quad \varepsilon= \pm 1, k \in(p), t \in(q)
$$

Then by virtue of (1.20) and (1.21), we get

$$
\eta^{\alpha}\left(\xi_{\beta}\right)=\delta_{\beta}^{\alpha}
$$

and

$$
\phi^{2}=a^{2}\left\{e^{k} \otimes e_{k}+\bar{e}^{t} \otimes \bar{e}_{t}\right\}
$$

or

$$
\phi^{2}=a^{2}\left\{I-\eta^{\alpha} \otimes \xi_{\alpha}\right\}
$$

Thus $M$ admits an unified contact structure

$$
\left(\phi, \xi_{\alpha}, \eta^{\alpha}\right)_{\alpha \in(r)}
$$

Hence the proof. 
2. On unified contact manifold $M$, with structure $\sum=\left(\phi, \xi_{\alpha}, \eta^{\alpha}\right)_{\alpha \in(r)}$

We define two operators

$$
P=I \otimes I-\phi_{1} \otimes \phi_{1}-\phi_{2} \otimes \phi_{2}-\phi_{3} \otimes \phi_{3}
$$

and

$$
Q=\phi_{1} \otimes \phi_{1}+\phi_{2} \otimes \phi_{2}+\phi_{3} \otimes \phi_{3}
$$

with properties

$$
P+Q=. I, \quad P^{2}=P, \quad Q^{2}=Q, \quad P Q=Q P=0 .
$$

Lemma 2. [2]. If $A$ is a projection operators, i.e. $A^{2}=A$ and $B=I-A$, then all the solutions of the equation $A x=y$ are of the form $x=y+B w$, where $w$ is orbitrary.

Definition. A distribution $D$ on a manifold $M$ is said to be parallel with respect to a given connection $\Gamma$. If, for every vector fields $Y$ and $X$ which belongs to the distribution $D$, the vector field $\nabla_{Y} X$ belongs to the distribution $D$, where $\nabla$ is covariant derivative with respect to the connection $\Gamma$.

Proposition 1. [3]. The distribution $D^{+}$given by (1.7) is parallel with respect to a connection $\Gamma$, iff

$$
\nabla \phi_{1} \circ \phi_{1} \doteq 0
$$
iff

Proposition 2. [3]. The distribution $D^{-}$is parallel with respect to a connection $\Gamma$

$$
\nabla \phi_{2} \circ \phi_{2}=0
$$
iff

Proposition 3. [3]. The distribution $D^{0}$ is parallel with respect to a connection $\Gamma$

$$
\nabla \phi_{3} \circ \phi_{3}=0
$$

Now if we assume that $\nabla$ is an $\sum$ - connection, then from (1.10), (1.11), (1.12) we get $\nabla \phi_{1}=0, \nabla \phi_{2}=0, \nabla \phi_{3} \doteq 0$.

Theorem 2. If $\Gamma$ is an $\sum$-connection on an unified contact manifold $M$, then the distribution $D^{+}, D^{-}, D^{0}$ given by (1.7), (1.8) and (1.9) are parallel with respect to this connection.

Proof. By virtue of proposition 1, 2, and 3 we obtain the required proof.

Now we shall find all connections. of the form

$$
\bar{\nabla}_{X}=\nabla_{X}+A_{x}
$$


with respect to which the distributions $D^{+}, D^{-}, D^{0}$ given by (1.7), (1.8) and (1.9) are parallel, where $\nabla$ is the co-derivative with respect to any orbitrary connection $\Gamma$ on an unified contact manifold $M$ and $A$ is a tensor field of type (1,2), with $A_{X} Y=A(X, Y)$.

Now for any vector $Y$ and for any tensor field $f$ of type (1.1)

$$
\begin{aligned}
\left(\dot{\bar{\nabla}}_{X} f\right)(Y) & =\bar{\nabla}_{X}(f Y)-f\left(\bar{\nabla}_{X} Y\right) \\
& =\nabla_{X}(f Y)+A_{X} f Y-f \nabla_{X} Y-f A_{X} Y \\
\bar{\nabla}_{X} f & =\left(\nabla_{X} f\right) Y+A_{X} \circ f-f \circ A_{X} .
\end{aligned}
$$

Now suppose that the distributions $D^{+}, D^{-}, D^{0}$ are parallel with respect to $\bar{\nabla}$, from proposition 1, 2 and 3, [3] we get

$$
\bar{\nabla}_{X} \phi_{1} \circ \phi_{1}=0, \quad \bar{\nabla}_{X} \phi_{2} \circ \phi_{2}=0, \quad \bar{\nabla}_{X} \phi_{3} \circ \phi_{3}=0 .
$$

Now by virtue of (2.8) and (2.9) we have

$$
\begin{aligned}
& \nabla_{X} \phi_{1} \circ \phi_{1}+A_{X} \phi_{1}^{2}-\phi_{1} A_{X} \phi_{1}=0, \\
& \nabla_{X} \phi_{2} \circ \phi_{2}+A_{X} \phi_{2}^{2}-\phi_{2} A_{X} \phi_{2}=0, \\
& \nabla_{X} \phi_{3} \circ \phi_{3}+A_{X} \phi_{3}^{2}-\phi_{3} A_{X} \phi_{3}=0 .
\end{aligned}
$$

Now adding these equations and making use of (1.13) and (1.14) we get

$$
\begin{aligned}
\nabla_{X} \phi_{1} \circ \phi_{1} & +\nabla_{X} \phi_{2} \circ \phi_{2}+\nabla_{X} \phi_{3} \circ \phi_{3}+A_{X} \\
& -\phi_{1} A_{X} \phi_{1}-\phi_{2} A_{X} \phi_{2}-\phi_{3} A_{X} \phi_{3}=0 .
\end{aligned}
$$

Using (2.2) we get

$$
\nabla_{X} \phi_{1} \circ \phi_{1}+\nabla_{X} \phi_{2} \circ \phi_{2}+\nabla_{X} \phi_{3} \circ \phi_{3}+P A_{X}=0 .
$$

This equation is equivalent to

$$
P A_{X}=\phi_{1} \nabla_{X} \phi_{1}+\phi_{2} \nabla \phi_{2}+\phi_{3} \nabla_{X} \phi_{3} .
$$

Hence, in virtue of lemma 2, we obtain

$$
A_{X}=\phi_{1} \nabla_{X} \phi_{1}+\phi_{2} \nabla_{X} \phi_{2}+\phi_{3} \nabla_{X} \phi_{3}+Q S_{X}
$$

where $S_{X}$ is an orbitrary tensor field of type $(1,2)$ with $S_{X} Y=S(X, Y)$. Thus we have,

Theorem 3. If, on an unified contact manifold $M$ with a structure $\sum=$ $\left(\phi, \xi_{\alpha}, \eta^{\alpha}\right)_{\alpha \in(r)}$ there exists any linear connection $\Gamma$ then distributions $D^{+}, D^{-}, D^{0}$ given by (1.7), (1.8), (1.9) respectively are parallel with respect to every connection $\Gamma$ given by

$$
\bar{\nabla}_{X}=\nabla_{X}+\phi_{1} \nabla_{X} \phi_{1}+\phi_{2} \nabla_{X} \phi_{2}+\phi_{3} \nabla_{X} \phi_{3}+Q S_{X}
$$


3. An unified Contact Riemannian manifold of $P$-Sasakian type [4]

Suppose that $M$ is an unified contact Riemannian manifold with a structure $\sum=$ $\left(\phi, \xi_{\alpha}, \eta^{\alpha}, g\right)_{\alpha \in(r)}$. We define the following tensor fields of type (2.2).

$$
\begin{aligned}
F=\frac{1}{2}\{I \otimes I & +\eta^{\alpha} \otimes \xi_{\alpha} \otimes I+I \otimes \eta^{\alpha} \otimes \xi_{\alpha} \\
& \left.-\eta^{\alpha} \otimes \xi_{\alpha} \otimes \eta^{\beta} \otimes \xi_{\beta}-\frac{1}{a^{2}} \phi \otimes \phi\right\}
\end{aligned}
$$

and

$$
\begin{aligned}
H= & \frac{1}{2}\left\{I \otimes I-\eta^{\alpha} \otimes \xi_{\alpha} \otimes I-I \otimes \eta^{\alpha} \otimes \xi_{\alpha}\right. \\
& \left.+\eta^{\alpha} \otimes \xi_{\alpha} \otimes \eta^{\beta} \otimes \xi_{\beta}+\frac{1}{a^{2}} \phi \otimes \phi\right\}
\end{aligned}
$$

with properties

$$
F+H=I \otimes I, \quad H H=H, \quad F F=F, \quad F H=H F=0
$$

we introduce

$$
L=H-\frac{1}{a^{2}} \phi \otimes \phi
$$

Now we define a symmetric tensor field $\phi$ of type $(0,2)$ as follows:

$$
\phi(X, Y)=g(\phi X, Y)
$$

Definition. An unified contact Riemannian manifold $M$, with a structure $\sum=$ $\left(\phi, \xi_{\alpha}, \eta^{\alpha}, g\right)_{\alpha \in(r)}$ is said to be of unified contact type of the following condition is satisfied:

$$
2 \phi(X, Y)=\left(\nabla_{X} \eta^{\alpha}\right) Y+\left(\nabla_{Y} \eta^{\alpha}\right) X \quad \text { for all } \quad \alpha \in(\mathrm{r})
$$

If, moreover, all $\eta^{\alpha}$ are closed, then since $d \eta^{\alpha}=0$ is equivalent to $\left(\nabla_{X} \eta^{\alpha}\right) Y=$ $\left(\nabla_{Y} \eta^{\alpha}\right) X$, the condition (3.5) is reduced to

$$
\phi(X, Y)=\left(\nabla_{X} \eta^{\alpha}\right) Y, \quad \text { for all } \quad \alpha \in(\mathrm{r})
$$

From $\eta^{\alpha}(X)=g\left(X, \xi_{\alpha}\right), \alpha \in(r)$ we have

$$
\left(\nabla_{X} \eta^{\alpha}\right) Y=g\left(\nabla_{X} \xi_{\alpha}, Y\right)
$$

and then (3.6) is equivalent to

$$
\phi X=\nabla_{X} \xi_{\alpha} \quad \text { for all } \quad \alpha \in(\mathrm{r})
$$


now we prove the following.

Theorem 4. Let $M$ be an unified contact Riemannian manifold of unified contact type with structure $\left(\phi, \xi_{\alpha}, \eta_{\alpha \in(r)}^{\alpha}\right.$. If

(i) all $\eta^{\alpha}$ are closed, and

(ii) The tensor field $\phi$ satisfies the condition $L \nabla_{Z} \phi=0$.

Then

$$
\begin{aligned}
\nabla_{Z} \phi(X, Y)=-a^{2} \sum_{\alpha} \eta^{\alpha}(X)\left[g(Y, Z)-\sum_{\beta} \eta^{\beta}(Y) \eta^{\beta}(Z)\right] \\
-a^{2} \sum_{\alpha} \eta^{\alpha}(Y)\left[g(X, Z)-\sum_{\beta} \eta^{\beta}(X) \eta^{\beta}(Z)\right]
\end{aligned}
$$

Proof. Since $\eta^{\alpha}$ are closed then (3.6) is satisfied, we have

$$
\begin{aligned}
\phi(\phi X, Y) & =a^{2}\left[g(X, Y)-\sum_{\alpha} \eta^{\alpha}(X) \eta^{\alpha}(Y)\right] \\
\nabla_{Z} \phi(X, Y) & =g\left(\left(\nabla_{Z} \phi\right) X, Y\right)
\end{aligned}
$$

From (3.9), we have

$$
\begin{aligned}
\nabla_{Z} \phi(\phi X, Y)= & a^{2}\left\{\nabla_{Z} g(X, Y)-\left(\nabla_{Z} \eta^{\alpha}\right)(X) \sum_{\alpha} \eta^{\alpha}(Y)\right. \\
& \left.-\left(\nabla_{Z} \eta^{\alpha}\right)(Y) \sum_{\alpha} \eta^{\alpha}(X)\right\}
\end{aligned}
$$

Putting $\phi_{Y}$ instead of $Y$ into (3.11), and making use of (3.6), we get

$$
\begin{aligned}
\nabla_{Z} \phi(\phi X, \phi Y)= & a^{2}\left\{-\nabla_{Z} \phi(X, Y)-\sum_{\alpha} \eta^{\alpha}(Y) \phi(Z, X)\right. \\
& \left.-\sum_{\alpha} \eta^{\alpha}(X) \phi(Z, \phi Y)\right\}
\end{aligned}
$$

From (3.6) and (3.10), we have

$$
\nabla_{Z} \phi\left(X, \xi_{\alpha}\right)=g\left(\left(\nabla_{Z} \phi\left(X, \xi_{\alpha}\right)=-g\left(\nabla_{Z} \xi_{\alpha}, \phi X\right)=-\phi(Z, \phi X)\right.\right.
$$

and

$$
\nabla_{Z} \phi\left(\xi_{\alpha}, \xi_{\alpha}\right)=0
$$

The condition (ii) is equivalent

$$
\begin{aligned}
0= & \left(L \nabla_{Z} \phi\right)(X, Y) \\
& -\frac{1}{2}\left\{\nabla_{Z} \phi(X, Y)-\nabla_{Z} \phi\left(\eta^{\alpha}(X) \xi_{\alpha}, Y\right)-\nabla_{Z} \phi\left(X, \eta^{\alpha}(Y) \xi_{\alpha}\right)\right. \\
& \left.+\nabla_{Z} \phi\left(\eta^{\alpha}(X) \xi_{\alpha}, \eta^{\beta}(Y) \xi_{\beta}\right)-\frac{1}{a^{2}} \nabla_{Z} \phi(\phi X, \phi Y)\right\} \\
= & \frac{1}{2}\left\{\nabla_{Z} \phi(X, Y)-\eta^{\alpha}(X) \nabla_{Z} \phi\left(\xi_{\alpha}, Y\right)-\eta^{\alpha}(Y) \nabla_{Z} \phi\left(X, \xi_{\alpha}\right)\right. \\
& \left.+\eta^{\alpha}(X) \eta^{\beta}(Y) \nabla_{Z} \phi\left(\xi_{\alpha}, \xi_{\beta}\right)-\frac{1}{a^{2}} \nabla_{Z} \phi(\phi X, \phi Y)\right\}
\end{aligned}
$$


On account of (3.12), (3.13) and (3.14) we have

$$
\nabla_{Z} \phi(X, Y)=-\sum_{\alpha} \eta^{\alpha}(Y) \phi(Z, \phi X)-\sum_{\alpha} \eta^{\alpha}(X) \phi(Z, \phi Y)
$$

Now using (3.9) in (3.15), we get

$$
\begin{aligned}
\nabla_{Z} \phi(X, Y)= & -a^{2} \sum_{\alpha} \eta^{\alpha}(Y)\left[g(X, Z)-\sum_{\beta} \eta^{\beta}(X) \eta^{\beta}(Z)\right] \\
& -a^{2} \sum_{\alpha} \eta^{\alpha}(X)\left[g(Y, Z)-\sum_{\beta} \eta^{\beta}(Y) \eta^{\beta}(Z)\right]
\end{aligned}
$$

Hence the theorem.

Definition. An unifined contact Riemannian manifold $M$, with structure

$$
\sum=\left(\phi, \xi_{\alpha}, \eta^{\alpha}, g\right)_{\alpha \in(r)}
$$

satisfying the conditions (3.6) and (3.8) is said to be of P-Sasakian type.

\section{References}

[1] A. Bucki and A.Meirnowski, "Almost r-paracontact structure," Am. Univ. Mariae curieskioduwska, Sec. A, 39 (1985).

[2] M.Obata, "Hermitian manifolds with quatemian structures," Tohoku Maih. J. 10(1958), 11-18.

[3] Andrzej Bucki, "Spacial connections on almost r-paracontact manifolds," J. Nigemian Math. Society. Volume 3, 1984, 27-33.

[4] Andrzej Bucki, "Almost r-paracontact structures of P-Sasakian type," Tensor, N.S. vol. 42 (1985) 42-54.

Department of Mathematics, Banaras Hnudu University, Varan'si 221005, India. 